Vol. 5, No. 1, 2020

\title{
THE IMPACT OF MICROWAVE RADIATION IN THE PROCESSES OF CARBON DIOXIDE ABSORPTION BY CHLOROPHYLL-PRODUCING MICROALGAE
}

\author{
Vasyl Dyachok, Solomiia Mandryk, Serhiy Huhlych \\ Lviv Polytechnic National University, \\ Department of Ecology and Sustainable, Environmental Management, \\ 12, S. Bandery Str., Lviv, 79013, Ukraine \\ dyachokvasil@gmail.com,solomiia.mandryk@ukr.net, zvit.reagent@gmail.com
}

https://doi.org/10.23939/ep2020.01.014

Received: 20.12.2019

(C) Dyachok V., Mandryk S., Huhlych S., 2020

\begin{abstract}
The influence of microwave radiation on the rate of $\mathrm{CO}_{2}$ uptake by chlorophyll-producing microalgae of the Chlorella Vulgaris has been established. Experimental dependencies of greenhouse gases absorption by microalgae depending on the impact of microwave electromagnetic (MEM) field have been obtained. The mathematical model of the dynamics of biomass growth of Chlorella Vulgaris microalgae depending on the time of electromagnetic radiation has been constructed. Based on the mathematical model solution and the experimental data obtained, a chart of the dependence of $\mathrm{CO}_{2}$ absorption by microalgae of the Chlorella Vulgaris provided that microwave radiation is present, has been constructed. The optimal value of microwave electromagnetic radiation for the design of technological schemes for the industrial purification of gas emissions from carbon dioxide by biological method has been established.
\end{abstract}

Key words: photosynthesis, electromagnetic radiation, Chlorella Vulgaris, biomass dynamics, mathematical model, kinetics.

\section{Introduction}

We live in the age of excessive electromagnetic radiation (EMR). In nature, the sources of electromagnetic fields are atmospheric electricity, cosmic rays, and radiation of the sun. Anthropogenic sources include generators, transformers, antennas, microwave ovens, mobile communication, refrigeration equipment, induction cookers, computer monitors and etc.. All of them have a negative impact on the human body.

Microwave radiation is a powerful physical irritant. All living organisms have different sensitivity to natural and anthropogenic (artificial) sources of microwave radiation: the nature and severity of the biological effect depend on the parameters of microwave radiation and the level of organization of the biosystem. Millimeter waves mainly affect the receptor apparatus, and longer wavelengths affect the central nervous system. Due to the action of electromagnetic radiation, the development of cataract, mental disorder, increased fatigue and drowsiness, the appearance of auditory hallucinations are observed. Prolonged exposure to radiation disrupts the functions of the cardiovascular system, impairs metabolism, and leads to changes in blood composition, reducing biochemical activity.

Numerous biological studies have shown that organisms of different species - from unicellular to human - are sensitive to permanent electromagnetic radiation. Today, much data is collected to demonstrate the stimulatory, inhibitory, or destructive effects of this radiation on microbial objects. According to many scientists, the biological effect of electromagnetic radiation is caused by the effect on water molecules, substances dissolved in it and the state of cell membranes. The ability to inhibit the development of microorganisms has all types of EMR, relatively weaker and more energy-intensive. Today, quite a lot of data have been collected to demonstrate the mutagenic nature of EMR: single-stranded DNA breaks and increased chromosomal aberrations. Significant inhibition of DNA synthesis and repair processes under the influence of weak electromagnetic fields $(50-100 \mathrm{~Hz})$ is also shown. [1]

Along with the action of inhibitory nature, there are examples of stimulation of certain processes of life of microorganisms. Electromagnetic radiation in the 
millimeter frequency range induces the formation of active intracellular metabolites. Microwave EMR (900 $\mathrm{MHz}$ ) has no mutagenic effects on the yeast Saccharomyces Cerevisiae, and higher frequency fields in the $50-55 \mathrm{GHz}$ range even have stimulating effects [1]. There is a lot of information in the literature on the effect of electromagnetic radiation on multicellular organisms [2], but little information is available on the effect of it on unicellular organisms, such as chlorophyll-producing microalgae Chlorella Vulgaris.

In this regard, it is important to investigate the effect of electromagnetic radiation on the growth of chlorophyll-producing microalgae Chlorella Vulgaris.

The purpose of the study is to study the effect of electromagnetic radiation on the rate of $\mathrm{CO}_{2}$ uptake by chlorophyll-producing microalgae Chlorella Vulgaris in an aqueous medium.

\section{Theoretical part}

It is believed that under the influence of electromagnetic radiation the rate of diffusion through biological membranes, the orientation, and conformation of biological macromolecules may change, as well as the state of the electronic structure of free radicals. Apparently, the mechanisms of biological action of the electromagnetic field are mainly nonspecific in nature and are associated with changes in the activity of the body's regulatory systems.

There are two types of influence of electromagnetic radiation on biological objects: thermal action (induced currents in tissues); specific action (resonant energy absorption by protein molecules, direct and non-direct effect on the central nervous system, ionization and polarization of molecules.

The effect of EMR on biological objects is estimated by the amount of electromagnetic energy (W) that will be absorbed by the object when it is in the field:

$$
W=\sigma \cdot S
$$

where $\sigma$ is the flux density of electromagnetic energy, W $/ \mathrm{m}^{2} ; \mathrm{S}$ is an effective absorbent surface, $\mathrm{m}^{2}$.

Due to the absorption of energy by the body of EMR, the tissue is heated, the higher the field strength and the longer the exposure time. The process of interaction of EMR with living organisms can be presented in phases. The first phase is the absorption of EMR energy by living cells in the form of a rupture of intermolecular bonds and the destruction of DNA. Phase 2 is the transformation of the EMR energy into chemical energy with the formation of ions and free active radicals. The third phase is the development of radiochemical reactions in the irradiated living organism [3].
Free radicals are compounds with free electrons. Free radicals can be occupied by electrons of any substance, most often oxygen. In this case, oxygen can no longer circulate in the body and provide respiration. Free radicals have a high reactivity, that is why they destroy cells and tissue walls. Therefore, free radicals are extremely harmful to any living organism. EMR stimulates their formation.

The initial link in the development of biochemical transformations under the influence of EMR is the primary inactivation (deprivation of activity) of enzymes (major catalysts and metabolism stimulants) by free radicals. In fats, chain oxidation reactions occur under the influence of radicals which are accompanied by the release of free fatty acids with toxic properties. The action of EMR leads to the breakdown of nucleotide molecules, proteins that are part of DNA, inhibit synthesis and activation of protein breakdown. In other words, EMR disrupts metabolism - the main process for maintaining life in the body. Metabolism is accompanied by the release of special substances - histamine (a strong allergen), hemolysin (disrupting blood cells), various toxins. These biochemical disorders occur inside and outside the cells and lead to morphological changes (from the Greek. Morphe - "form"): various deformations, disturbance of the structure and appearance of cells and tissues. The extent of all of the above disorders depends on the radiosensitivity of cells and tissues: the higher the radiosensitivity, the greater the damage from EMR. Particularly sensitive to EMR are organs where active cell reproduction occurs. Against the background of electromagnetic effects, the internal environment in the body changes due to the accumulation of various toxins (the process of toxemia). Toxemia leads to further destruction of cells and tissues and impaired regulation. Neuroendocrine changes play a significant role in shaping the mechanism of adaptation of a living organism to environmental conditions and ensure the relative constancy of the internal environment of the organism. When exposed to EMR on the body, this stability is disrupted by neuroendocrine "breakdowns".

To study the effect of electromagnetic radiation on microorganisms, we chose the green microalgae culture Chlorella Vulgaris. Microscopic chlorophyll-producing microalgae Chlorella Vulgaris are unpretentious in their habitat and are capable of intensive reproduction, which is why they are found everywhere: in freshwater bodies, seas, and soils. They absorb dozens of times more carbon dioxide compared to terrestrial plants. This state of affairs makes it promising to use microalgae in the technologies of biological treatment of gas emissions from greenhouse gases. 


\section{Presentation of the main material and discussion of the results}

In the course of the experimental study, the object of study was the culture of green microalgae - Chlorella Vulgaris. Experimental studies were conducted in photobioreactors at certain values of microwave electromagnetic (MEM) radiation of microalgae (Fig. 2).

For this study, a culture of microalgae - Chlorella Vulgaris was added into an aqueous solution with a standard nutrient medium. It has been cultured for 8 days in six photobioreactors, with a volume of $1 \mathrm{dm}^{3}$. Nutrients - carbon dioxide and mineral nutrients of the cell, microalgae are obtained directly from the environment of the liquid medium, absorbing them with their surface. At the time of this study, the temperature was maintained within $\mathrm{t}=30^{\circ} \mathrm{C}$ to achieve the most favourable cultivation conditions. Besides, research hardware provided sufficient carbon dioxide throughout the photobioreactor volume. The organization of mixing and illumination by solar energy contributes to the intensification of the processes of carbon dioxide absorption, which was accompanied by changes in the number of microalgae cells (biomass). A complex series of biochemical reactions organized in a certain way in space and time are the basis for the processes of cell exchange with the environment and internal metabolism. As a result of these transformations, the dynamics of growth of microalgae biomass in suspensions and other quantities change. A mathematical model describing the dependence of the change in cell concentration in the cultivation medium on time $\mathrm{N}=\mathrm{N}(\mathrm{dt})$ is given in [4].

The selection of algae biomass has been carried out for eight days, with a fixed interval of days. Determination of changes in the dynamics of growth of biomass microalgae in suspension was carried out by a photocolorimetric method. For this purpose, a calibration line was constructed, using the method of measuring the optical density of previously known values of microalgae density in the solution. (Fig. 1). According to the data obtained, a calibration graph was constructed.

Using the constructed calibration graph, the change in the dynamics of microalgae cell biomass growth in the suspension was determined, since the obtained line is described by an equation the slope angle of which is $\mathrm{k}=0,2274$; and the coefficient of determination $\mathrm{R}^{2}=0,9916$.

Based on the coefficient $\mathrm{k}$ obtained from the calibration graph (Fig. 1), the change in the optical density of microalgae Chlorella Vulgaris growth was subsequently transferred to a change of biomass dynamics.

To study the effect of microwave radiation on the growth of chlorophyll-producing microalgae, the first sample has been irradiated for 15 seconds, the second for
30 seconds, the third for 1 minute, the fourth for 2 minutes, and the fifth for 3 minutes.

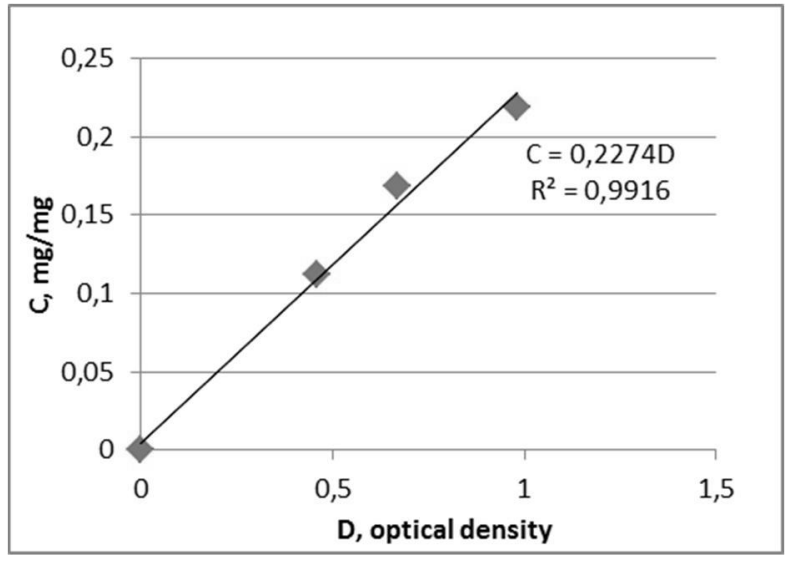

Fig. 1. Calibration line of determining the changes in the dynamics of the microalgae biomass in the suspension by a photocolorimetric method.

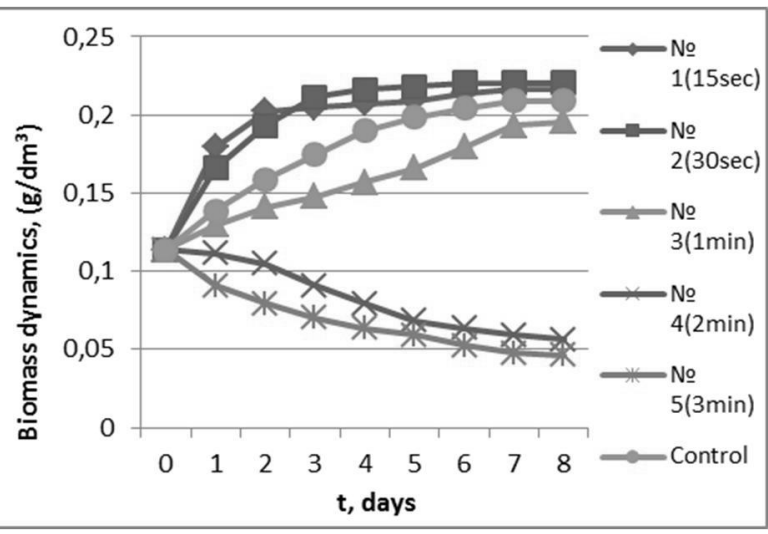

Fig. 2. Dynamics of the microalgae biomass growth at certain values of MEM radiation.

Based on the experimental data and the calculated values, graphical dependencies in the changes of microalgae biomass dynamics on the time at certain single values of the MEM radiation in the cultivation medium were obtained (Fig. 2).

Analyzing the data (Fig. 2), it should be noted that change in the dynamics of microalgae cell biomass growth is significantly dependent on the energy of MEM radiation compared to the control that was not exposed to MEM. Thus, the increase in biomass microalgae in a well-mixed cultivation medium changes over time depending on the energy of the MEM radiation.

At low MEM energy, there is a slight increase in microalgae cells - activation, but to a certain value. As can be seen in (Fig. 2), in the first and second photobioreactors, there is a slight increase which is even higher on the $5^{\text {th }}$ day than in the control sample, but from the sixth day, we see an alignment with the control. At the same time, there is a decrease in the dynamics of 
microalgae cell biomass growth compared to the control in other photobioreactors with much higher values of MEM energy. This is indicated by a detrimental effect on microalgae cells or the occurrence of inactivation.

One of the known inactivation theories states that MEM radiation emits inactivation of microorganisms solely by heating. Damage to membranes, processes of denaturation of enzymes, proteins, nucleic acids, and other vital components occur in the same way as under normal heating.

According to the other mechanism, there are nonthermal effects of the inactivation of microorganisms. These include the appearance of pores in the membrane under the influence of the potential difference on its surface; rupture of cell membranes under the influence of this potential difference; cell dissolution, which is due to the fact that some critical molecules inside the cell, absorbing the energy of the MEM field, change and destroy the internal components of the cell of microalgae.

There is no consensus as to which of the mechanisms is actually present under the MEM radiation. The research data do not separately support any mechanism. This can be explained by the difficulty of comparing conventional and microwave heating since the heating conditions are generally not the same (microwave heating is faster). The distribution of temperatures inside the suspension of microalgae under the two mechanisms of heating is different (for microwave heating, the temperature on the surface of the suspension is lower than in the suspension of microalgae, and under traditional heating - on the contrary). Under the conditions of MEM radiation, selective heating occurs, which is absent in the process of traditional heating. That is, due to the different electrical properties of the components of the suspension of microalgae heating is faster.

In a stationary mode, the MEM energy of the field entering the experimental chamber from the magnetron should be equal to the energy absorbed by the product, in our case the suspension of chlorophyll-producing microalgae (losses in the camera walls and other types of losses are neglected). Therefore, the energy absorbed by algae is determined by the formula:

$$
W=p \times V=2 \pi v \times \varepsilon_{0} \times \varepsilon^{\prime \prime} \times E^{2} \times V,
$$

where $V$ is the volume of the product under study; $p$ is $a$ specific power.

If at constant values $(W=$ const $)$ and type of test sample $\left(\varepsilon^{\prime \prime}=\right.$ const $)$ the volume of the test product $V$ is decreased, then the field strength $E$ in the placement of this product decreases inversely with the square root of the volume.

This dependence makes it easy to adjust the intensity of the field by changing the volume of the test specimen. For this purpose, ballast (water) was installed into the photobioreactor under study with specified parameters. Therefore, it became possible to adjust the field intensity in the photobioreactor by changing the ballast volume.

If the volume subjected to heating is sufficiently small, then the intensity of the field will exceed $\left(\varepsilon^{\prime \prime} \times V\right)$. Therefore, the mass is selected as a control parameter (easy to measure and control) for further research.

The ability of the suspension or solution to interact with the MEM field is characterized by its complex dielectric constant:

$$
\varepsilon=\varepsilon^{\prime}+i \varepsilon^{\prime \prime}
$$

where $\varepsilon^{\prime}$ is the dielectric constant that characterizes the product's ability to store the MEM field energy, and $\varepsilon$ " is the loss factor that determines the substance's ability to absorb the MEM field energy and convert it into heat.

Although it is difficult to separate the dielectric and conductivity losses, the loss factor is divided into two components:

$$
\varepsilon^{\prime \prime}=\varepsilon^{\prime \prime}+\frac{\sigma}{\varepsilon_{0} \omega}
$$

where $\sigma$ is the conductivity of the sample under study. Other parameters are often used to characterize losses instead of $\varepsilon^{\prime}: \sigma=\omega \times \varepsilon_{0} \times \varepsilon^{\prime \prime}$ is equivalent conductivity.

The dielectric constant $\varepsilon$ allows us to determine other constants that characterize the distribution of the field in space, in particular, $\delta p$ is the depth of penetration by power; $\delta$ is the depth of penetration by tension and $\lambda$ is the wavelength in the experimental sample.

For a given frequency $v=2450 \mathrm{MHz}$ :

$$
\lambda=\frac{c}{v}=\frac{3 \times 10^{8}}{245 \times 10^{7}}=0.122 \mathrm{M}=122 \mathrm{MM}
$$

For most suspensions and solutions of biological media, water is $90 \%$ to $95 \%$ (even milk contains $90 \%$ of water), so for such tested products, the effect of each small ingredient on the complex dielectric constant $\varepsilon$ does not depend on the presence or absence of other small ingredients. Accordingly, it can be written as:

$$
\varepsilon=\varepsilon_{0}+\sum_{k} \varepsilon_{k},
$$

where $\varepsilon_{0}$ is the complex constant for the main ingredient, and $\varepsilon_{k}$ is the corrections made by the small ingredients.

Since the concentrations of small ingredients $c_{k}$ are small, the dependence of the corrections $\varepsilon_{k}$ on the concentration of $c_{k}$ can be considered linear, since, for the values $c_{k}=0$, the correction $\varepsilon_{k}$ must also correspond to the zero value, which is confirmed by the experimental data (Fig. 1).

Consequently, the heat released into a dielectric contained in a microwave electromagnetic field depends on its complex dielectric constant $\varepsilon$ of formula (3) and knowing the specific thermal power (power released per unit volume) from formula (2)

$$
p=2 \pi v \times \varepsilon_{0} \times \varepsilon^{\prime \prime} \times E^{2},
$$


where

$$
E=\varepsilon^{\prime} \times E_{0},
$$

$E_{0}$ is the field strength in vacuum. These formulas calculate a spatially homogeneous unbounded dielectric.

In our case, the dielectric is a two-phase system. We call one of the phases the medium and mark the quantities that refer to it by index 2, and the other inclusions and the corresponding quantities are denoted by index 1 . In this case, the field near the inclusions and in the middle of the inclusion is distorted, that is, not determined by formula (8). We assume that the concentration of the inclusions is so small that the distortions of the magnetic field caused by the individual inclusions are not superimposed on each other and, accordingly, the electromagnetic field strength in the medium away from the inclusions $\mathrm{E}_{2}$ is determined by formula (8). In this case, the MEM field strength inside the inclusions will be determined by the formula:

$$
E_{1}=\gamma \times E_{2},
$$

$\gamma$ is a coefficient that depends on the shape of the inclusions. In particular, for spherical form inclusions:

$$
\gamma=\frac{3 \varepsilon_{1}^{\prime}}{\varepsilon_{2}^{\prime}+2 \varepsilon_{1}^{\prime}} .
$$

The obtained results of measurements of complex dielectric constant showed [5] that for some microorganisms considered as inclusions and culture medium $\varepsilon^{\prime \prime} 1>\varepsilon^{\prime \prime} 2$. From this, it follows that the specific power released in the microalgae is greater than that released in the environment, therefore, the microalgae heat more than the cultivation medium. This is the phenomenon of selective heating in the MEM field of chlorophyll-producing microalgae.

As confirmed by the experimental data (Fig. 3), changes in the dynamics of the microalgae biomass growth depend on the energy of microwave radiation in comparison with the control. As can be seen from Fig.3, the obtained radiation energies of more than $3 \mathrm{~kJ}$ decrease the biomass dynamics of microalgae in the photobioreactor.

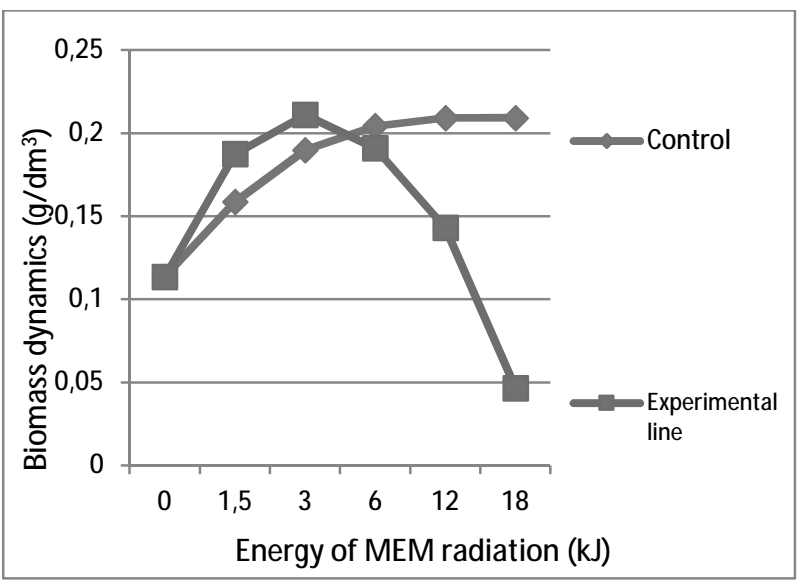

Fig. 3. Change in the microalgae biomass dynamics under the influence of MEM radiation.
If we talk not about lethality, but about changes in microalgae under the influence of MEM radiation, many authors report that such changes under the influence of low power radiation do not lead to a significant change in the temperature of microalgae [6]. The process may depend on the parameters of the MEM radiation - power, exposure time, continuous or pulsed. The mechanism of inactivation may also depend on the type of microalgae. Thus, emphasis should be placed on the detrimental effects of MEM radiation on living organisms, in particular on microalgae. But it should be carefully used in the process of cleaning gas emissions from greenhouse gases, among which carbon dioxide is the most important, to say the least.

\section{Conclusion}

This paper presents the results of the experimental studies of the dynamics of the microalgae biomass growth, depending on the energy of microwave radiation. Experimental dependencies of carbon dioxide absorption by microalgae depending on the impact of microwave electromagnetic radiation was constructed. A mathematical model of the dynamics of the growth of biomass of Chlorella Vulgaris microalgae depending on the time and energy of irradiation by the MEM field is presented. Based on the mathematical model and the experimental results obtained, it is possible to predict the equipment for the design of technological schemes for the purification of industrial gas emissions of carbon dioxide by Chlorella Vulgaris microalgae under the influence of microwave irradiation. A detrimental effect of MEM energy emission of more than $3 \mathrm{~kJ}$ on the growth of microalgae, and thus on the dynamics of carbon dioxide absorption, has been established.

\section{References}

[1] Goldovskaya L. F.: Himiya okruzhayuschey sredyi, 3-e izd. Mir,Moskva 2008. (in Russian)

[2] Zolotarova O. K., Shniukova Ye. I., Syvash O. O., Mykhailenko N. F.: Perspektyv. Vykorys. mikrovodorostei u biotekhnol.. Alterpres, Kyiv 2008. (in Ukrainian)

[3] Klap Ya. A., Yaremkevych O. S., Chervetsova V. H. et al.: Bull. Nat. Univ. "Lviv Polytechnic" ser.: "Chem., technol. of substan. and their appl.”,2016,841,168.(in Ukrainian)

[4] Dyachok V. V., Huhlych S. I., Levko O. B.: Bull. Nat. Univ. "Lviv Polytechnic" ser.: "Chem., technol. of substan. and their appl.", 2015, 812, 365. (in Ukrainian)

[5] Burdo O. G., Ryibina O. B.: Protses. Inaktivat. mikroorgan. v mikrovolnov. pole. Poligraf, Odessa 2010. (in Ukrainian)

[6] Dyachok V., Huhlych S., Yatchyshyn Y., Zaporochets Y., Katysheva V.: Chem. Chem. Technol., 2017,11,1,111. 